\title{
Challenges in Diagnostic and Systemic Treatment of Metastatic Lung Atypical Carcinoid: a Case Report
}

\author{
Vlad Mihai Croitoru ${ }^{1}$, Diana Bogdan ${ }^{1}$, Ioana Mihaela Dinu ${ }^{1}$, Monica lonela Miron¹,

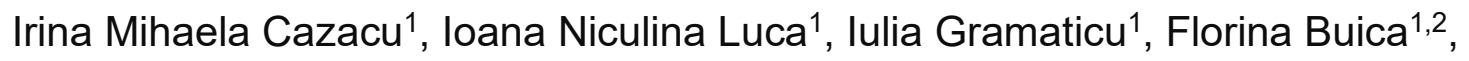 \\ Adina Emilia Croitoru ${ }^{1,2}$ \\ ${ }^{1}$ Department of Oncology, Fundeni Clinical Institute \\ ${ }^{2}$ Faculty of Medicine, "Titu Maiorescu" University Bucharest, Romania
}

Corresponding author: Diana Bogdan e-mail: diana_bogdan@yahoo.ro

\begin{abstract} Chemotherapy

\section{Introduction}

Neuroendocrine tumors (NETs) are rare neoplasms that originate from the diffuse neuroendocrine cell system and can be found in different sites of the body. Most commonly these tumors occur in the gastroenteropancreatic system (70\%), followed by NETs of the lung, thymus and other less
\end{abstract}

Neuroendocrine lung tumors are rare neoplasms, with an increasing incidence in the last thirty years. Metastatic disease is rarely curable. We present the case of a 65year-old male patient with atypical functional carcinoid of the lung and synchronous liver metastases beginning with carcinoid syndrome treated with somatostatin analogues, chemotherapy and peptide receptor radionuclide therapy, with a good clinical response and remission of the carcinoid syndrome.

Keywords: Atypical Lung Carcinoids, Somatostatin analogs, PRRT, Everolimus,

common sites such as hepato-biliary tract, ovaries and testes [1]. The incidence of lung NETs is low (25\%), although it has increased in the last 30 years, due to improved diagnostic methods [2].

NETs of the lung represent a group of tumors arising from neuroendocrine cells of the bronchopulmonary epithelium. According to the latest WHO classification, lung NETs 
include a miscellaneous population of tumors ranging from well-differentiated pulmonary NETs or typical carcinoids (TC) to intermediate grade or atypical carcinoids (AC) and poorly differentiated small-cell lung carcinoma (SCLC) and large cell neuroendocrine carcinoma (LCNEC) $[1,3]$. Well-differentiated NETs have the ability to produce hormones almost identical to those of the nervous system, but compared to gastroenteropancreatic NETs, lung carcinoids are rarely associated with hypersecretion and paraneoplastic syndromes. The carcinoid syndrome and Cushing's syndrome are most commonly found in carcinoids and are rare for patients with LCNECs or SCLCs [2].

Somatostatin analogues (SSAs), chemotherapy and peptide receptor radionuclide therapy (PRRT remain the most effective options available based on specific tumor features. Also, the treatment perspective in the management of advanced lung carcinoids increases after the recent success of everolimus in improving progression free survival (PFS). However, the best sequence of these therapies have not yet been defined [4].

\section{Case presentation}

A 65-year-old man with a medical history of arterial hypertension and diabetes mellitus type 2, presented in August 2016 with flushed skin, abdominal pain and diarrhea. Physical exam revealed facial erythema and hepatomegaly. The blood tests showed a slight elevation of fibrinogen and C-reactive protein. The colonoscopy and upper gastrointestinal endoscopy did not show any significant abnormalities. An abdominal magnetic resonance imaging (MRI) showed hepatic steatosis and multiple liver masses of variable enhancement and sizes, ranging from a few millimetres to $4 \mathrm{~cm}$ diameter (Fig. 1A).

The chest CT scan revealed a tumor mass of $2.1 \mathrm{~cm} \times 1.5 \mathrm{~cm}$ in the superior Fowler segment of the right lung, highly suggestive of malignancy; right hilar lymphadenopathy of $2 \mathrm{~cm} \times 2 \mathrm{~cm}$ and also an osteocondensation of L1 vertebrae (Fig. 1B). A bronchoscopy was performed, but the biopsy revealed no malignant cells.

The case was discussed in the multidisciplinary meeting and a CT guided liver biopsy was indicated for diagnosis confirmation. The histopathological report showed an epithelial proliferation with neuroendocrine differentiation; immunohistochemistry staining was positive for chromogranin and synaptophysin and proliferation index was 10 mitosis per $2 \mathrm{~mm}^{2}$ ((ten high-power fields(10HPF)). Urine measurement of 5hydroxyindoleacetic acid ((5-HIIA 4.8 $\mathrm{mg} / 24 \mathrm{~h}$; normal value (NV): 2-7 mg/24h)) and serum chromogranin $A$ was elevated: $766 \mathrm{ng} / \mathrm{l}$ (NV: 27-94 ng/L). Therefore, the diagnosis was metastatic lung NET with carcinoid syndrome.

We initiated SSA therapy (octreotide LAR 30mg every 28 days) with good clinical response and decreased neuroendocrine tumor markers. The first follow-up imaging evaluation (3 months after therapy) showed stable disease. However, a 1-year follow-up CT scan performed revealed the 
progression of liver metastases, along with an elevation of chromogranin A (950 ng/l) and urinary 5-HIIA (7.8 mg/24h). The octreoscan evaluation showed intense radiotracer uptake in the lung and liver metastases (Fig. 2A). Therefore, an add-on therapy with Capecitabine at 600 $\mathrm{mg} / \mathrm{m}^{2}$ orally bd on days 1-14 and TMZ (Temozolomide) $150 \mathrm{mg} / \mathrm{m}^{2}$ bd on days $10-$ 14 of a 28-day cycle was initiated.

Until July 2019, clinical status of the patient and follow-up CT-scans showed stable disease, although tumour markers continued to grow (urinary 5-HIIA 26.4 mg/24h; chromogranin A $8580 \mathrm{ng} / \mathrm{l})$. Octreoscan evaluation (Fig. 2B), showed progression of the lung tumor and liver and bone metastases with positive somatostatin
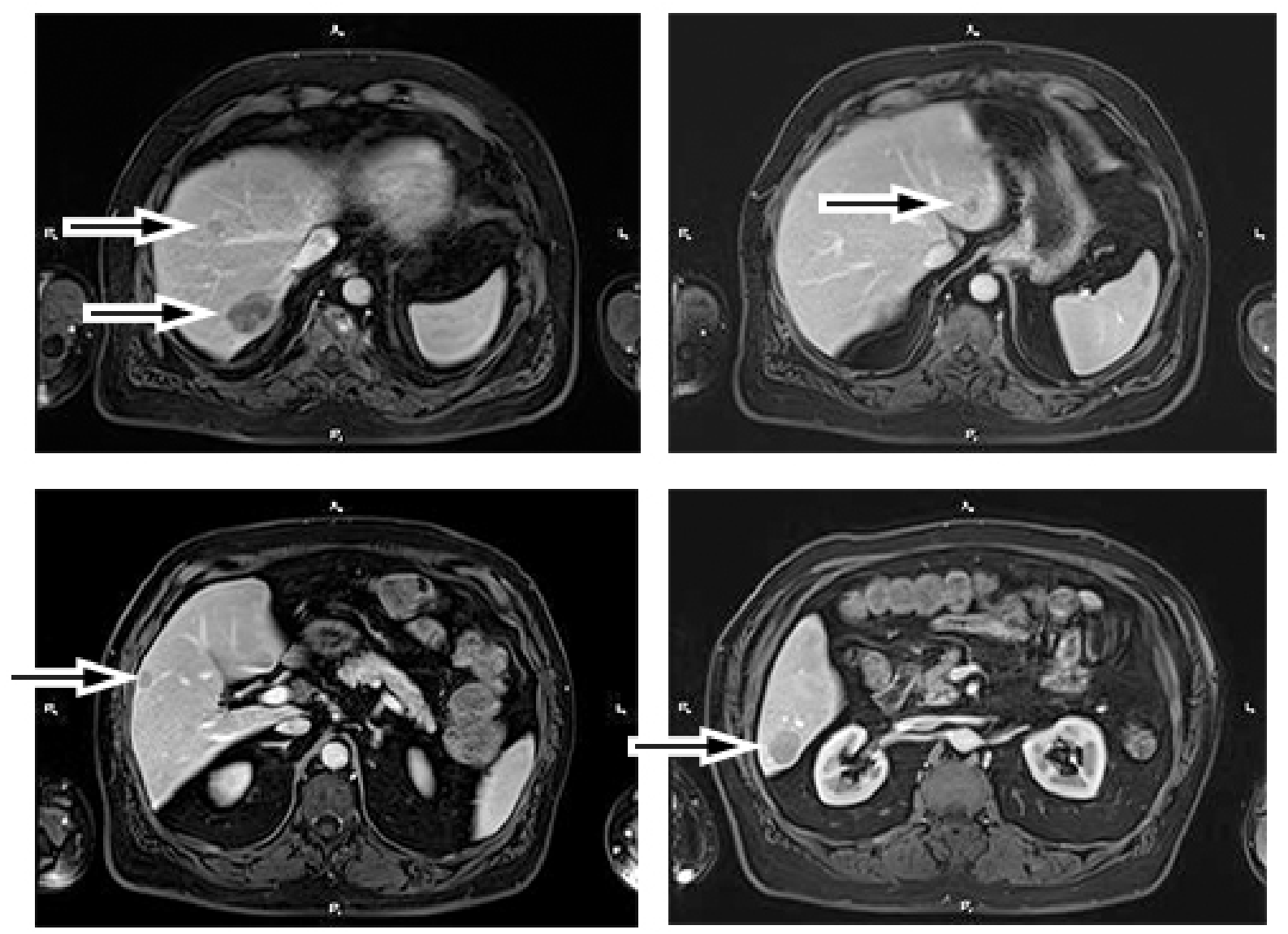

Fig. 1A. Abdominal MRI (Aug 2016): a well-defined liver lesion of $4 \times 3 \mathrm{~cm}$, heterogeneous and variable attenuation and other smaller hypervascular lesions. 


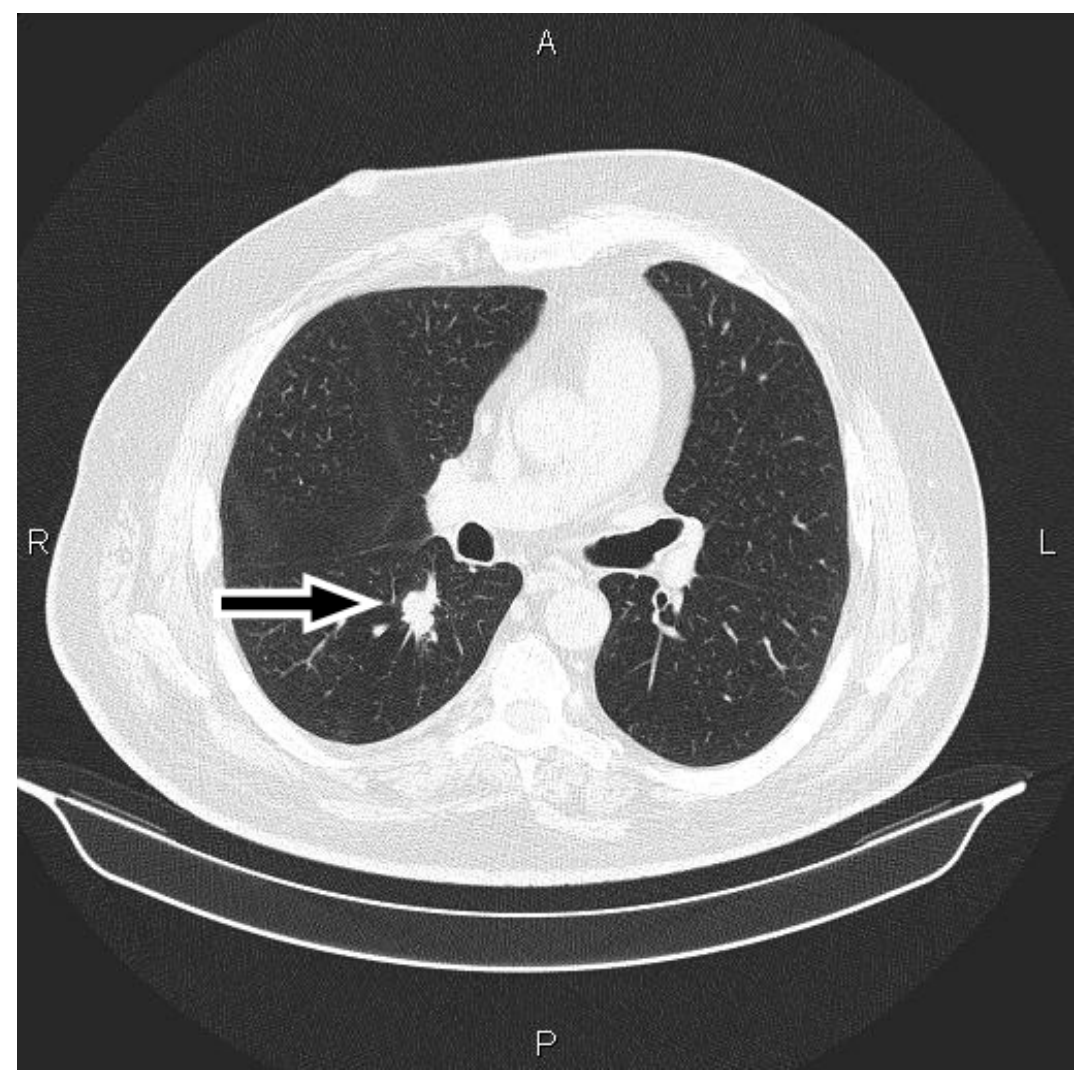

Fig. 1B. Thorax CT scan (Aug 2016): a spiculated and irregular nodule in the Fowler segment of the right lung.
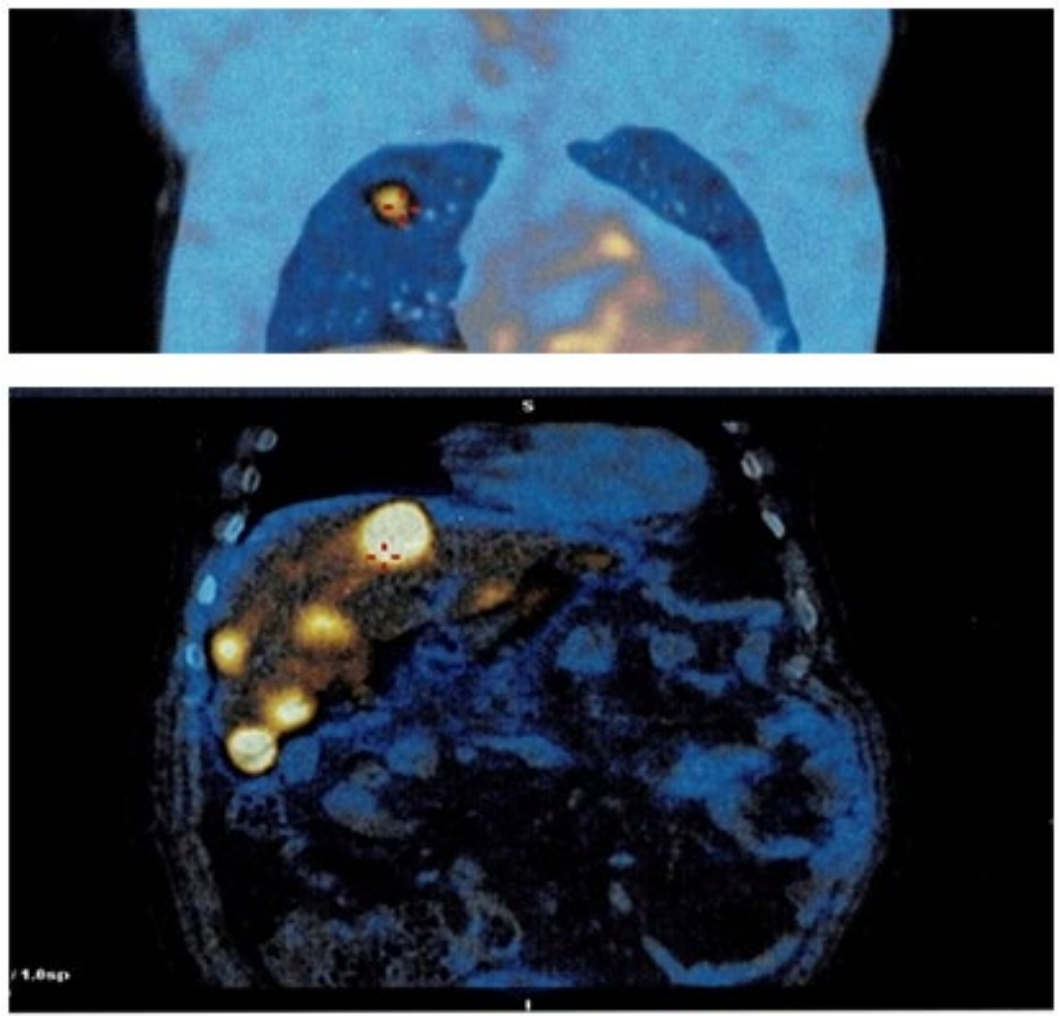

Fig. 2A. Somatostatin receptor scintigraphy (Sep 2017): multiple intense focal uptakes in the lung and liver indicating somatostatin receptor positive lung tumor and liver metastasis. 


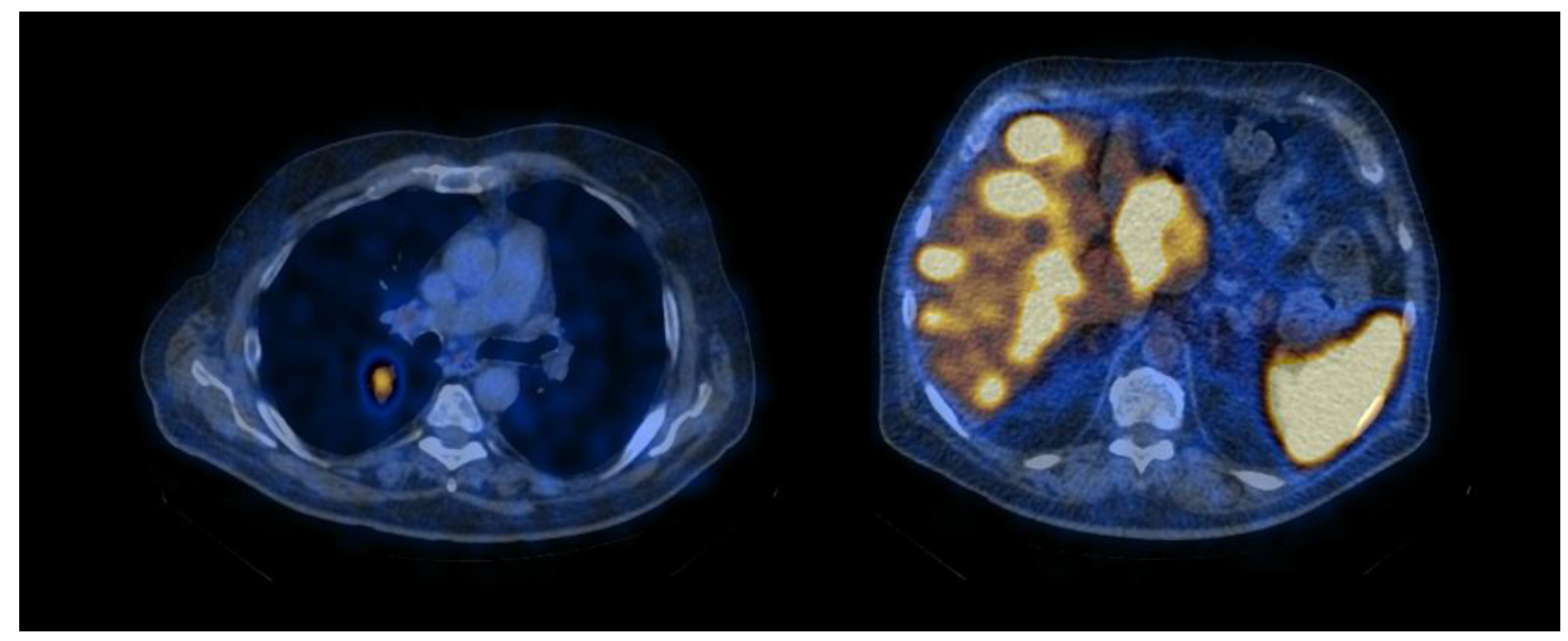

Fig. 2B. Jul 2019, Octreoscan with Tc-99m tektrotyd labeled somatostatin analogues: progression of the lung tumor and liver metastases.

\section{Discussion}

The incidence of lung neuroendocrine tumors has increased significantly in the past three decades due to available diagnostic methods. These tumors represent about 1$2 \%$ of all lung tumors and $20-30 \%$ of all NETs. About $70 \%$ of all broncho-pulmonary NETs are located in the major bronchi and the rest in the periphery of the lungs. They occur in almost $60 \%$ of the cases in the right lung, such as in our case, and particularly in the middle lobe $[5,6]$.

About $90 \%$ of the patients with central bronchial NETs present with symptoms such as hemoptysis, cough, recurrent pulmonary infections, fever, chest discomfort and unilateral wheezing, while peripheral carcinoids are incidentally discovered in most of the cases. The occurrence of a carcinoid syndrome or Cushing's syndrome are very rare in patients with pulmonary NETs [7]. In the case presented, the patient had carcinoid syndrome without any respiratory symptoms.
Patients suspected of having lung NETs, based on biomarkers or clinical symptoms, should undergo confirmation of diagnosis with histopathological examination and immunohistochemistry; radiologic assessment is required to determine the extent of the disease.

The diagnosis of neuroendocrine phenotype and the correct NET classification should include the evaluation of specific neuroendocrine markers. Among these, chromogranin A and synaptophysin expression are the most specific stains. The sensitivity and specificity of chromogranin $A$ for the detection of NETs are between $70 \%$ and $100 \%$ [8]. In this patient's case, a close correlation between the increase in chromogranin $\mathrm{A}$ values and the progression of the disease was observed. Other markers that may be helpful to define a neuroendocrine phenotype include PGP 9.5, NSE and CD56. Additionally, the proliferation index $\mathrm{Ki}-67$ immunostaining is also an extremely useful marker to a better classification of the lung NETs [9]. 
The increasing number of diagnostic procedures and therapeutic options require a multidisciplinary approach and decisiontaking in multidisciplinary tumor meetings to ensure a personalized treatment approach, as we did for our patient. Bronchoscopy with biopsies and endoscopic ultrasonography if necessary, is the best procedure to detect central bronchial NETs. In our case, because the bronchoscopy with biopsy was negative for malignant cells, a liver biopsy from the metastases was performed in order to identify tumor pathology [7].

The purpose of treatment in metastatic lung NETs is prolongation of survival and palliation of symptoms and is mainly based on systemic therapies. Currently, there are no data to support a specific sequence of the following systemic therapy options for metastatic disease: SSAs (octreotide LAR and lanreotide Autogel/Depot); PRRT (177Lu-DOTATATE/90-Y-DOTATOC), chemotherapy (Platinum + Etoposide or Temozolomide based regimens) and targeted therapy (everolimus). SSAs represent a classic treatment for malignant carcinoid syndrome and are frequently included in the therapeutic management of NETs. According to preclinical and clinical studies of the last 20 years, SSAs have dual inhibitory action, against hormone secretion and proliferation of cells, although they do not generally shrink tumors [10]. Consequently, because of the onset of the disease with carcinoid syndrome, our patient's treatment was initiated with octreotide LAR
$30 \mathrm{mg} / \mathrm{month}$, with a good clinical and biological response.

A randomized, double-blind, phase 3 study (SPINET, NCT02683941) evaluating the efficacy and safety of lanreotide-autogel vs. placebo is ongoing in patients with welldifferentiated, metastatic and/or unresectable [11]. ATLANT (NCT02698410) is a phase 2 , multicentre, single arm, open-label trial with the purpose to assess efficacy and safety of the combination of lanreotide with temozolomide $(250 \mathrm{mg} /$ day for 5 consecutive days every 28 days for a maximum of 48 weeks) in thoracic well differentiated NET [12]. Mechanisms of resistance at SSA include mutation of SSTR2 or SSTR5 and signalling defect [13]. Concomitant administration of SSAs with other molecules might represent a promising strategy to avoid or delay the development of drug resistance. Among targeted agents, there is a strong rationale for combining SSAs with everolimus or with metronomic chemotherapy [14].

A significant improvement in the management of progressive advanced lung NETs has been made with the introduction of everolimus (mTOR inhibitor) in clinical practice. Systemic treatment with everolimus received FDA approval in 2016 based upon RADIANT-4, a randomized, double-blind, placebo-controlled, phase 3 trial. RADIANT4 met its primary endpoints, indicating significant increase in median PFS (11 and 3.9 months in everolimus and placebo arms) $[15,16]$. Unfortunately, everolimus was not available for our patient, because 
at that time it was not approved in our country in the metastatic pulmonary NET setting.

PRRT represents a type of systemic radiotherapy that uses radiolabelled SSAs to target neoplastic cells that express SSRs. ${ }^{177}$ Lu-DOTATATE and ${ }^{90} \mathrm{Y}$-DOTATOC are the two representative types of PRRT and contain Lutetium-177 and Yttrium-90, respectively. PRRT may be considered in cases of progressive lung carcinoids with intense SSR expression. According to current NCCN guidelines, treatment with 177-Lu-DOTATATE is indicated for patients with broncho-pulmonary NETs, SSR positive imaging and disease progression on SSAs, if the tumor is either low grade (typical) with clinically significant tumor burden, or intermediate grade (atypical) [16]. Imhof et al. [17] conducted one of the largest studies evaluating response, survival and safety profile of an Yttrium-90 labelled radiopeptide in 1109 patients with 25 different neuroendocrine cancer subtypes including 84 lung carcinoids. In the subgroup of lung carcinoids, RR was $28.6 \%$ with no complete responses. Median survival was 2.9 times longer than the expected value detected for G1-G2 advanced NENs with a longer survival correlated to high tumor baseline uptake and morphological, biochemical and clinical response. Two recent studies have evaluated the role of PRRT in a homogeneous population of lung carcinoids. In the first study [18], 114 patients with advanced bronchopulmonary carcinoid treated in a referral centre with three different PRRT protocols ([Yttrium-90DOTATOC vs [Lutetium-177-DOTATATE vs [Yttrium-90-DOTATOC + [Lutetium-177-

DOTATATE) were retrospectively evaluated. The estimated median OS and PFS were 58.8 and 28.0 months, respectively. The cohort of 21 patients treated with a combination of both radiopeptides obtained the highest ORR and PFS rates at 3 years after the start of PRRT (38.1\% and $46.2 \%$, respectively), while treatment with [Lutetium-177DOTATATE was associated with the highest 5-year OS (61.4\%) and the lowest rate of adverse events. In the second study, 34 consecutive patients with progressive advanced lung carcinoids treated with four or five cycles of [Lutetium-177-DOTATATE were prospectively evaluated. PRRT achieved 15\% RR in the entire population with 3\% complete response (all TCs), while median PFS and OS were 18.5 and 48.6 months, respectively. Regarding the histological type, TCs obtained a higher percentage of disease control rate (DCR) ( $80 \%$ vs $47 \%)$ and better survival in terms of PFS (20.1 vs 15.7 months) as well as OS (48.6 vs 37.0 months) [19].

Lung carcinoids are generally tumors with low proliferative capacity and cytotoxic chemotherapy has a limited activity. However, chemotherapy remains an option for a palliative setting in metastatic lung NETs. Several chemotherapeutic drugs either alone or in combination have been tested in metastatic disease: 5-fluorouracil (5-FU), capecitabine, doxorubicin, dacarbazine, 
streptozotocin, cyclophosphamide, platinum derivatives, etoposide and temozolomide, but all these drugs showed a modest overall response rate $(<30 \%)$ with low efficacy when administered in various combinations [20]. Etoposide-platinum based regimens represent the treatment of choice in poorly differentiated lung NETs (SCLC, LCNELC). Another attractive chemotherapeutic agent in the management of lung carcinoids is Temozolomide in monotherapy or in combination. For patients with clinically significant bronchopulmonary tumor burden that is low or intermediate grade, temozolomide alone or in combination with octreotide or lanreotide is an option to manage tumor burden or any associated symptoms [21]. Treatment with capecitabine and temozolomide (CAPTEM) has shown significant activity in patients with pancreatic NETs with reported objective response rates ranging from $33 \%$ to $70 \%$ and median PFS ranging from 18 months to 22.7 months, respectively [22]. Moreover, the combination of CAPTEM demonstrated superior PFS and OS compared with temozolomide monotherapy in a randomized prospective study of patients with progressive pancreatic NETs, thereby establishing CAPTEM as a standard of care in this disease. Temozolomide monotherapy has shown modest activity in lung NENs. In a retrospective study of 31 lung NETs (including 14 TCs and 15 ACs), a partial response (PR) was observed in three patients $(14 \%)$, and the median PFS was 5.3 months [23]. To our knowledge, there are no published data on the use of CAPTEM in lung NENs.

\section{Conclusions}

Lung NETs are rare neuroendocrine neoplasms. The prognosis of NETs depends on the tumor grade and on the extent of the disease. Treatment recommendations for patients with bronchopulmonary NETs require multidisciplinary team and should be individualized. The main purpose of treatment for advanced or metastatic NETs of the lung is mainly to palliate symptoms. SSAs, everolimus, chemotherapy and PRRT therapy remain the most effective options. Randomized trials are required to better define the optimal therapeutic sequence. Many other targeted agents are currently evaluated in clinical trials on pulmonary NETs and significant results may emerge in the future.

\section{Abbreviations}

AC - Atypical carcinoids

DCR - Disease control rate

HPF - High-power fields

LCNEC - Large cell neuroendocrine carcinoma

$\mathrm{MRI}$ - Magnetic resonance imaging 
NETs - Neuroendocrine tumors

OS - Overall survival

PFS - Progression free survival

PRRT - Peptide receptor radionuclide therapy

SCLC - Small-cell lung carcinoma

SSAs - Somatostatin analogues

SSR - Somatostatin receptors

TC - Typical carcinoids

WHO - World Health Organization

\section{Statements:}

Authors' contributions: DB, VMC, IMC and AEC conceived the analysis. DB, VMC and AEC collected the data and performed the analysis. DB, AEC, IMD and MIM participated in the treatment of the patient.

Consent for publication: As the corresponding author, I confirm that the manuscript has been read and approved for submission by all co-authors.

Conflict of interest: All authors declare having no competing interests associated with this publication.

Funding Sources: This research did not receive any specific grant from funding agencies in the public, commercial, or not-for-profit sector.

Informed Consent: The informed consent was obtained from the patient for publication and any accompanying images.

Statement of Ethics: The accompanying manuscript does not contain any studies carried out by the authors on humans or animals.

Ethical Approval: The treatment strategy/study protocol was approved by a local tumor board.

\section{References:}

1. Lloyd R, Osamura R, Klöppel G, Rosai J. WHO Classification of Tumours of Endocrine Organs, 4th edn, vol. 10. IARC Press, Lyon; 2017.

2. Rekhtman N. Neuroendocrine tumors of the lung: an update. Archives of Pathology and Laboratory Medicine. 2010;134(11):1628-38. 
3. Pelosi G, Sonzogni A, Harari S, Albini A, Bresaola E, Marchiò C, et al. Classification of pulmonary neuroendocrine tumors: new insights. Translational Lung Cancer Research. 2017;6(5):513.

4. Tsoukalas N, Baxevanos P, Aravantinou-Fatorou E, Tolia M, Galanopoulos M, Tsapakidis K, et al. Advances on systemic treatment for lung neuroendocrine neoplasms. Annals of translational medicine. 2018;6(8).

5. Gustafsson BI, Kidd M, Chan A, Malfertheiner MV, Modlin IM. Bronchopulmonary neuroendocrine tumors. Cancer. 2008;113(1):5-21.

6. Righi L, Volante M, Rapa I, Scagliotti GV, Papotti M. Neuro-endocrine tumours of the lung. A review of relevant pathological and molecular data. Virchows Archiv. 2007;451(1):51-9.

7. Öberg K, Hellman P, Ferolla P, Papotti M, Group EGW. Neuroendocrine bronchial and thymic tumors: ESMO Clinical Practice Guidelines for diagnosis, treatment and follow-up. Annals of Oncology. 2012;23(suppl_7):vii120-vii3.

8. Nehar D, Lombard-Bohas C, Olivieri S, Claustrat B, Chayvialle JA, Penes MC, et al. Interest of Chromogranin A for diagnosis and follow-up of endocrine tumours. Clinical endocrinology. 2004;60(5):644-52.

9. Righi L, Gatti G, Volante M, Papotti M. Lung neuroendocrine tumors: pathological characteristics. Journal of thoracic disease. 2017;9(Suppl 15):S1442.

10. Aparicio T, Ducreux M, Baudin E, Sabourin J-C, De Baere T, Mitry E, et al. Antitumour activity of somatostatin analogues in progressive metastatic neuroendocrine tumours. European Journal of Cancer. 2001;37(8):1014-9.

11. Efficacy and Safety of Lanreotide Autogel/ Depot $120 \mathrm{mg}$ vs. Placebo in Subjects With Lung Neuroendocrine Tumors (SPINET) 2020 [Available from: https://clinicaltrials.gov/ct2/show/NCT02683941.

12. (ATLANT) EaSoLAAiCWTiSWTNT. [Available from: https://www.clinicaltrials.gov/ct2/show/NCT02698410.

13. Theodoropoulou M, Stalla GK. Somatostatin receptors: from signaling to clinical practice. Front Neuroendocrinol. 2013;34(3):228-52.

14. Koumarianou A, Antoniou S, Kanakis G, Economopoulos N, Rontogianni D, Ntavatzikos A, et al. Combination treatment with metronomic temozolomide, bevacizumab and long-acting octreotide for malignant neuroendocrine tumours. Endocr Relat Cancer. 2012;19(1):L1-L4.

15. Yao JC, Fazio N, Singh S, Buzzoni R, Carnaghi C, Wolin E, et al. Everolimus for the treatment of advanced, non-functional neuroendocrine tumours of the lung or gastrointestinal tract (RADIANT-4): a randomised, placebo-controlled, phase 3 study. The Lancet. 2016;387(10022):968-77.

16. Network NCC. NCCN Clinical Practice Guidelines in Oncology: Neuroendocrine and Adrenal Tumors. Version 1.2019. 2019.

17. Imhof A, Brunner $\mathrm{P}$, Marincek N, Briel M, Schindler $\mathrm{C}$, Rasch $\mathrm{H}$, et al. Response, survival, and long-term toxicity after therapy with the radiolabeled somatostatin analogue [90Y-DOTA]-TOC in metastasized neuroendocrine cancers. J Clin Oncol. 2011;29(17):2416-23.

18. Mariniello A, Bodei L, Tinelli C, Baio SM, Gilardi L, Colandrea M, et al. Long-term results of PRRT in advanced bronchopulmonary carcinoid. Eur J Nucl Med Mol Imaging. 2016;43(3):441-52.

19. Ianniello A, Sansovini M, Severi S, Nicolini S, Grana CM, Massri K, et al. Peptide receptor radionuclide therapy with 177 Lu-DOTATATE in advanced bronchial carcinoids: prognostic role of thyroid transcription factor 1 and 18 F-FDG PET. European journal of nuclear medicine and molecular imaging. 2016;43(6):1040-6.

20. Forde PM, Hooker CM, Boikos SA, Petrini I, Giaccone G, Rudin CM, et al. Systemic therapy, clinical outcomes, and overall survival in locally advanced or metastatic pulmonary carcinoid: a brief report. Journal of Thoracic Oncology. 2014;9(3):414-8.

21. Chong CR, Wirth LJ, Nishino M, Chen AB, Sholl LM, Kulke MH, et al. Chemotherapy for locally advanced and metastatic pulmonary carcinoid tumors. Lung Cancer. 2014;86(2):241-6.

22. Al-Toubah T, Morse B, Strosberg J. Capecitabine and temozolomide in advanced lung neuroendocrine neoplasms. The Oncologist. 2020;25(1):e48.

23. Crona J, Fanola I, Lindholm DP, Antonodimitrakis P, Öberg K, Eriksson B, et al. Effect of temozolomide in patients with metastatic bronchial carcinoids. Neuroendocrinology. 2013;98(2):151-5. 